\title{
A Rare Cause of Headache in the Emergency Department: Intraventricular Epidermoid Cyst Rupture With Hydrocephalus
}

\author{
Mehmet Yigit ${ }^{\mathrm{a}, \mathrm{d}}$, Mehmet Hakan Seyithanoglu ${ }^{\mathrm{b}}$, Tolga Turan Dundar ${ }^{\mathrm{b}}$, Ozgur Sogut ${ }^{\mathrm{a}}$, \\ Eda Yigit ${ }^{\mathrm{c}}$
}

\begin{abstract}
Lateral intraventricular tumors are not frequently observed. Since these tumors grow linearly rather than exponentially, they grow gradually and thus do not cause mass effects and hydrocephalus. This study is the case report of a rare great volume left intraventricular epidermoid cyst rupture. The tumor was found to be associated with mass effect on neighboring structures and hydrocephalus.
\end{abstract}

Keywords: Epidermoid cyst; Rupture; Headache; Hydrocephalus

\section{Introduction}

Only $0.2-1 \%$ of all primary intracranial brain tumors are epidermoids $[1,2]$. In terms of histology, intracranial epidermoids are benign and they are congenital neoplasms of the central nervous system. Since these lesions grow slowly, they are usually seen in the fifth or sixth decades of life [2]. Cerebellopontine angle and suprasellar region are the most common locations where epidermoid tumors are located [3]. Intracranial epidermoid tumor is very rarely located in the lateral ventricle. The clinical presentation of intracranial epidermoid tumor is generally in the form of mental status alteration or headache.

When the literature was reviewed, only nine case reports of lateral ventricular epidermoid tumors were found. A great number of lateral intraventricular epidermoids are silent and/or do not produce hydrocephalus [2]. This study is a case report of a patient with unusual clinical presentation and pathological course who had a large intraventricular epidermoid tumor with

\footnotetext{
Manuscript accepted for publication May 10, 2016

aDepartment of Emergency Medicine, Haseki Training and Research Hospital, Istanbul, Turkey

bDepartment of Neurosurgery, Bezmialem Vakif University, Istanbul, Turkey 'Department of Emergency Medicine, Sisli Etfal Training and Research Hospital, Istanbul, Turkey

${ }^{\mathrm{d} C}$ Corresponding Author: Mehmet Yigit, Department of Emergency Medicine, Haseki Training and Research Hospital, Istanbul, Turkey.

Email: yigitacil@gmail.com
}

doi: http://dx.doi.org/10.14740/jocmr2585w a mass effect on the neighboring structures.

\section{Case Report}

A 43-year-old male patient was admitted with a complaint of moderate, gradually progressive headache and occasional episodes of vomiting that had been going on for 6 months. The patient's history did not suggest any neurological cause. Powers in the right upper limb and lower limb were $4 / 5$ and $3 / 5$, respectively. No other neurological deficit other than this was found. The patient's brain tomography showed lobulated contour image in the cerebrospinal fluid (CSF) density which formed a compression on the level of right lateral ventricular anterior horn of the left frontal region. The lesion had airfluid levels on the lateral ventricles and air particles on subarachnoid spaces. The rupture was evaluated in favor of cystic mass. The ventricular system had a dilated appearance (Fig. 1A).

Preoperative magnetic resonance imaging of the brain showed a lobulated, well-defined mass lesion in the anterior horn of the left lateral ventricle, which included hyperintense material hypointense on T1 (Fig. 1B) sequences and hypointense material hyperintense on T2 sequences (Fig. 1C). The lesion had a size of $30 \times 50 \mathrm{~mm}$, it had a lobulated contour and it was hyperintense. The lesion compressed the left lateral ventricle anterior horn and minimal periventricular ooze with obstructive hydrocephalus was observed on FLAIR sequences (Fig. 1D). The patient's lesion was completely removed with left frontal craniotomy, duraplasty and tisselian tissue adhesive. The tumor was avascular and pearly white and it covered the left lateral ventricle anterior horn. Postoperative contrast negative computed tomography showed near-total excision of the lesion and resolution of hydrocephalus. The patient's postoperative recovery was normal. Six months later, at his follow-up, the patient was found to have improvement in his hemiparesis as well.

The lesion was confirmed to be epidermoid as a result of histopathological examination. Parts of pearly white fragmented tissue were presented for formalin fixation and paraffin sectioning. The hematoxylin and eosin stained slide showed numerous anucleated squamous cells which did not show any presence of identifiable basal germinal cells. Skin adnexal tissues such as sebaceous glands, hair follicles or mucinous gland were observed. Overall features were consistent with epidermoid cyst. 


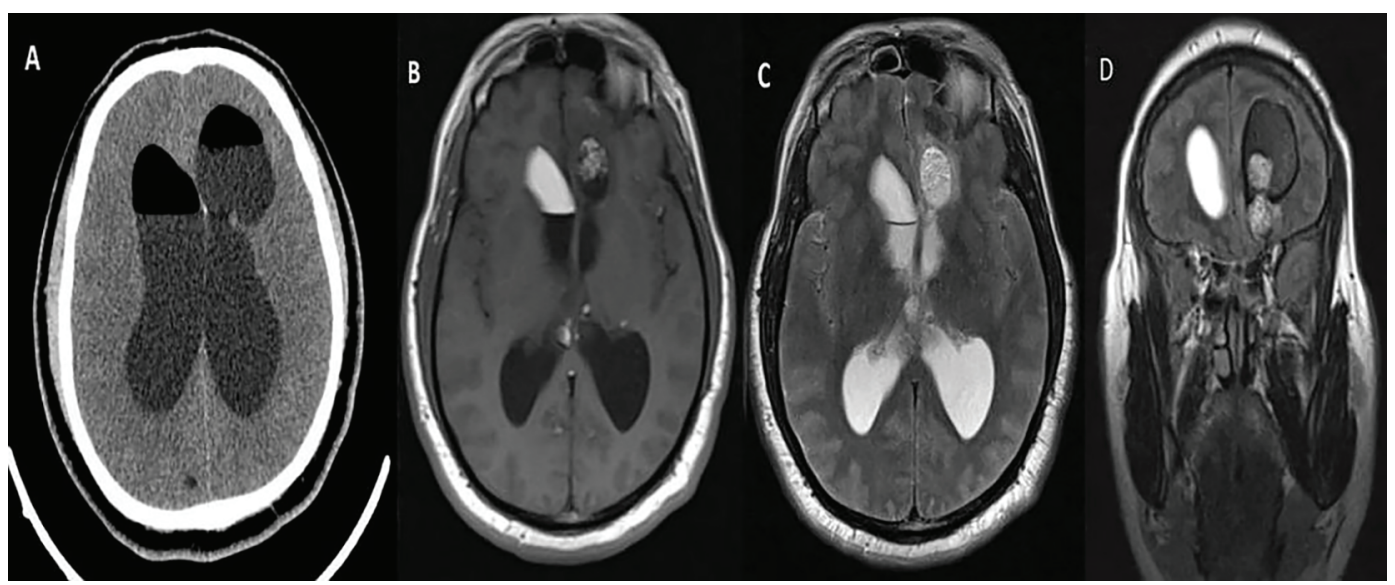

Figure 1. Axial CT imaging of the patient (A). Axial T1 (B), Axial T2 (C) and coronal FLAIR (D) magnetic resonance images of the patient.

\section{Discussion}

Only $0.2-1.0 \%$ of all intracranial tumors are epidermoid tumors [3]. Not only epidermoid tumors, but also lateral intraventricular tumors are rare [2]. Despite of advanced imaging tools, the number of epidermoid tumors and lateral intraventricular tumors remains low. When we searched the literature, we found only nine reported cases and no left lateralventricular cyst rupture case. Intraventricular epidermoid's pathogenesis is uncertain. However, a direct relation is thought to exist with the development of choroidal vessels which is an explanation of the lateral migration through the choroidal fissure and subsequent entrapment of neuroepithelial cells [4]. There is an association between median/paramedian structures and the tumors reported. Thus, the notion that these lesions originate from quadrigeminal cisterns needs to be seriously considered.

The accumulation of keratin and cholesterol which are the breakdown products of desquamated epithelial cells causes epidermoids to grow. Epidermoids are slow growing lesions since they grow linearly instead of exponentially. As a result of this slow growth, they are clinically seen in the later decades of life.

Most of the epidermoids do not present with hydrocephalus. This is because of the smooth and pliable nature of the cyst wall and its content, which allow progressive, slow molding of the surrounding neural structures. Moreover, it allows maintenance of CSF pathways [5]. Although lateral intraventricular epidermoid has no fixed pattern of clinical presentation since it is rare, it is possible to find some non-specific clinical patterns described in the literature such as headache, cognitive deficit or psychiatric symptoms.

Patients who have this type of lesion are usually in their fifties or sixties [4]. However, our case was 43 years old and this shows that although they grow slowly, these types of lesions can also be seen in the fourth decade of life. The patient in our study had hydrocephalus which resulted from compression of the lateral ventricle. The patient's hydrocephalus led to neurological deficit and headache, both of which improved after the lesion was removed with total microsurgery. This case is possibly the first one which has presented with rupture of the cyst and hydrocephalus with a rare type of lesion. Due to the symptomatic and clinical improvement observed in our patient, it can be said that microsurgical total removal is a better option to manage these types of lesions.

\section{Conclusion}

As a result, in patients who are thought to have cerebrovascular disease, especially those with a comorbid of ventricular hydrocephalus and spontaneous air presence, epidermoid cyst rupture should be considered.

\section{References}

1. Franko A, Holjar-Erlic I, Miletic D. Lateral ventricle epidermoid. Radiol Oncol. 2008;42:66-68.

2. Bhatoe HS, Mukherji JD, Dutta V. Epidermoid tumour of the lateral ventricle. Acta Neurochir (Wien). 2006;148(3):339-342; discussion 342.

3. Akar Z, Tanriover N, Tuzgen S, Kafadar AM, Kuday C. Surgical treatment of intracranial epidermoid tumors. Neurol Med Chir (Tokyo). 2003;43(6):275-280; discussion 281 .

4. Aher RB, Singh D, Singh H, Saran RK. Lateral intraventricular epidermoid in a child with hydrocephalus. J Pediatr Neurosci. 2012;7(3):205-207.

5. Kallmes DF, Gray L. Epidermoid and dermoid tumors: Imaging. In: Wilkins RH, Rengachary SS, editors. Neurosurgery. Vol. 1. New York: McGraw-Hill; 1995. pp. 965976. 